\title{
La catalogación de los dólmenes pirenaicos
}

\author{
E. Ripoll Perello *
}

La abundancia de monumentos megalíticos en la Europa Occidental suscitó muy pronto el interés por su catalogación. Esta inquietud nació a mediados del siglo xIX. Nos limitaremos a citar al respecto la siempre interesante obra de conjunto de James Fergusson, Rude Stone Monuments in all Countries, que pretendia abarcar un gran espacio geográfico ', y, para España, el trabajo pionero en tantos aspectos de Manuel de Góngora y Martínez sobre las antigüedades prehistóricas de Andalucia $^{2}$. En la presente nota pretendemos hacer una breve síntesis de la historia de dicha cuestión en las diversas regiones al norte y al sur de los Pirineos, dejando de lado los trabajos particularizados a uno o unos pocos monumentos, los referidos a los problemas del megalitismo en general y a las obras de sintesis, para referirnos casi exclusivamente a los intentos de inventario o catalogación.

Desde mediados del siglo xIx hubo interés en Francia por establecer la cantidad y la distribución geográfica de los monumentos megalíticos. La lista de aquellos primeros intentos fue trazada en 1901 por A. de

* Universidad Nacional de Educación a Distancia (UNED). Esta nota fue presentada al "Congreso Internacional de Historia de los Pirineos" celebrado en Cervera (Lérida) en el mes de noviembre de 1988 .

' James Fergusson, Rude Stone Monuments in all Countries, their Age and Uses, Londres, Johan Murray, 1872 (reproducción facsimil: Graz, Akademische Druck-u. Verlag. santalt, 1976).

2 Manuel DE Gongora y Martinez, Antigüedades prehistóricas de Andalucia, Madrid 1868 
Mortillet en un opúsculo sobre el tema ${ }^{3}$. En el recordaba los recuentos anteriores: "De la distribution des dolmens sur la surface de la France" de Alexandre Bertrand (Revue Archéologique, 1864); mapa del Essai sur les dolmens de A. de Boustetten (1965, 1875 y 1876); mapa de G. de Mortillet para la Nouvelle géographie universelle de Elisee Reclus (1877); mapa de A. de Mortillet para la traducción francesa de la obra de James Fergusson, Les monuments mégalithiques de tous pays (1878); los importantes inventarios de la "Sous-Commission des monuments mégalithiques", publicados en 1880 en los Bulletins de la Société d'Anthropologie de Paris; el mapa de A. de Mortillet publicado en el libro de Henri de Cleuzion, La création de l'homme et les premieres âges de l'humanite, y en el de Muston, Le préhistorique dans les pays de Montbeliard et les contrees circunvoisines (ambos de 1887). A. Bertrand recogía 2.273 dólmenes, número que A. de Mortillet llevaria a 4.226 en el año 1900. Las cifras para los departamentos pirenaicos era las siguientes: Pirineos Orientales, 26; Ariège, 15; Alto Garona, 0; Altos Pirineos. 12; y Bajos Pirineos, $19^{4}$.

En España, las iniciativas partieron de los grupos de investigadores del Pais Vasco, de Navarra y de Cataluña. En la primera de dichas regiones por su entidad y buen método para la época, hay que citar los trabajos zonales de Telesforo de Aranzadi -catedrático de la Universidad de Barcelona--, José Miguel de Barandiarán. Enrique de Eguren y $F$. de Ansoleaga ${ }^{5}$, o los más modernos y en forma de catálogo sistemático de J. Elosegui ${ }^{6}$. Como puente entre los focos dolménicos vascos y catalanes hay que recordar, para el Alto Aragón, las exploraciones juveniles de M. Almagro Basch ${ }^{7}$. En Cataluña hay que señalar los trabajos importantes de Luis Mariano Vidal, Manuel Cazurro, P. Bosch Gimpera, J. Rius Serra, J. Gudiol e I. Macau, y muy particularmente los libros de J. Serra Vilaró sobre la civilización megalítica de la Cataluña interior y el

A. DE MORTILlet, "Distribution geographique des dolmens et des menhirs en France", Revue de l'Ecole d'Anthropologie de Paris, XI. 1901. pags. 35-45 y 2 mapas. Una primera version de estos mapas habia sido preparada para ser exhibida en la Exposición Universal de 1900

* Datos tomados del citado articulo de A. de Mortillet.

Por ejemplo: Telesforo de ARanzadi, José Miguel de Barandiaran y Enrique de Egu REN. Exploración de dieciseis dólmenes de la Sierra de Elosua-Placentzia. San Sebastián 1922. y Exploración de seis dólmenes de la Sierra de Urbasa (Navarra). San Sebastián. 1923: T. de Aranzadi y F. DE Ansoleaga, Exploración de cinco dólmenes del Aralar. Pamplona 1915. y Exploración de catorce dólmenes del Aralar. Pamplona 1918.

J. Elosegul, Catálogo dolménico del Pais Vasco. San Sebastián, 1953

M. Almagro, "La cultura magalítica en el Alto Aragón". Ampurias, IV, 1942, págs. 155-169, y VI. 1944, págs. 311-316. 
intento de encuadre de Luis Pericot ${ }^{8}$. Este último, publicó en 1950 una nueva versión de su obra con otro título: Los sepulcros megalíticos catalanes y la cultura pirenaica ${ }^{9}$. Los trabajos modernos de M. Tarradell, A. Panyella, F. Riuró, J. Maluquer. P. de Paloi, J. Garriga y M. Oliva para la zona catalana, sumados a los que tenian lugar en el Pais Vasco por $\mathrm{J}$. Medrano y otros, aumentaron mucho el número de monumentos conocidos, siendo la correspondiente bibliografía fácilmente asequible. El estado de la cuestión en 1950 correspondía al mencionado libro de Pericot que recogía 211 dólmenes catalanes (más 46 dudosos), situados en un mapa junto con los del Rosellón (en número de 39). Aunque sin enumerarlos, también se establecía un repertorio de los sepulcros megalíticos vascos y del Alto Aragón y se prestaba atención a los del Ariège y del SO de Francia. Con todo ello, L. Pericot pudo trazar el mapa de distribución que aqui reproducimos (fig. 1).

En la década de los años 50, ante la avalancha de nuevos hallazgos y el desigual conocimiento de los antiguos, empezó a imponerse, incluso en ambientes internacionales, la necesidad de formar catálogos bien sistematizados de los monumentos miegalíticos. Sin duda en ello influyó la modélica labor llevada a cabo en el $\mathrm{S}$ de España por los esposos G. y $V$. Leisner ${ }^{10}$. Lo cierto es que en el entorno cientifico del profesor Luis Pericot, y concretamente en el Instituto de Prehistoria y Arqueología de la Diputación de Barcelona, se concentró la idea, a la que contribuimos, de lo que inmediatamente recibió el nombre de "Corpus de Sepulcros megalíticos". Se trataba de publicar el catálogo o inventario de los monumentos for comarcas naturales y con unas caracteristicas uriformes para las planimetrías, el dibujo de los materiales y la descripción tanto de los monumentos propiamente dichos como de los materiales. Además, se

"Luis Mariano VIDAL, Más monumentos megaliticos en Cataluña, Memorias de la Real Academia de Ciencias y Artes de Barcelona, 1894 (con anadidos en 1911). Manuel CAZU. RRO. Los monumentos megaliticos de la provincia de Gerona. Madrid. Centro de Estudios Históricos, 1912. P. Bosch Gimpera, Prehistoria catalana. Barcelona 1920. J. Rius Serra, "Sepuicres megalitics excavats pel Museu de Vich". Anuari de I'Institud d'Estudis Catalans, VI. 1915-1920. J. COLOMInES ROCA y Josep GUDIOL I RICART, Els sepulcres megalitics de IAuselania. Barcelona, 1923. Isidro MaCaU, "Nous monuments megalitics de l'Alt Emporda". Butll. de la Institució Catalana diHistoria Natural, núm. XXXIV. J. SERRA VILARO, La civilització megalítica a Catalunya, contribució al seu estudi, Solsona, 1926. Li. Pericot Garcia, Civilización megalitica catalana. Barcelona. Facultad de Filosofia y Letras, 1923 (que fue su tesis doctoral).

־ Luis Pericot Garcia, Los sepulcros megaliticos catalanes y la cultura pirenaica. Barcelona, Instituto de Estudios Pirenaicos. 1950

1. Georg y Vera LEISNER, Die Megalithgraber der Iberischen Halbinseln, 1, Die Sudden. Berlin 1943; 2. Der Westen. 1956 y 1959 (Madrider Forschungen. 1). 


$$
\text { E. RIPOLL PERELLO }
$$

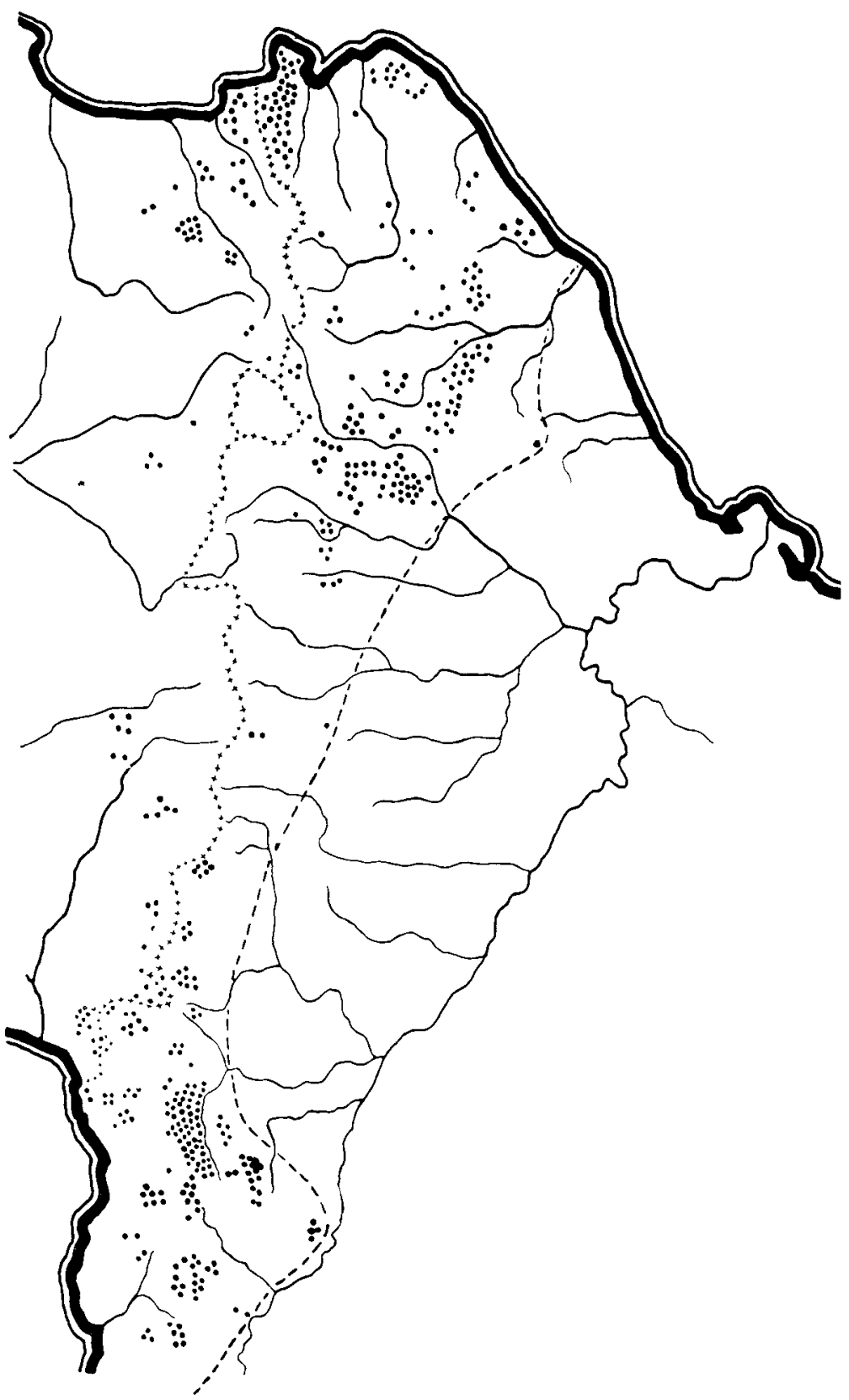

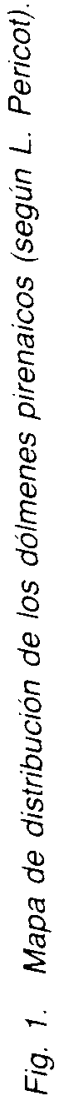

448 
invitaba a todos los centros de investigación a publicar los fasciculos de sus zonas de interés. El citado Instituto de la Diputación de Barcelona, el Servicio de Investigaciones de la Diputación de Gerona, el Departamento de Prehistoria del CSIC, y el Instituto de Arqueología de la Universidad de Barcelona, patrocinaron diferentes fasciculos. A tal iniciativa corresponden las nueve carpetas, con su correspondiente estudio, debidas a R. Batista Noguera, L. Esteva Cruañas, M. Cura Morera, A. M. Ferrán Ramis, J. Padró y J. Abelanet, referidas a las siguientes comarcas: Moianés ", Vic ${ }^{12}$, Les Gabarres ${ }^{13}$, Sierra de Roda en el Alt Empordà ${ }^{14}$, Vall de Cabó en el Alt Urgell ${ }^{15}$, Cerdanya y Capcir ${ }^{16}$, y Alt Empordà ${ }^{17}$.

Como puede verse en el mapa que acompañamos (fig. 2), el territorio que cubria el inventario catalán comprendia sólo un tercio aproximadamente del territorio de la parte oriental de los Pirineos. Pero que la empresa correspondia a una necesidad de la investigación prehistórica lo demuestra que por aquelias mismas fechas surgieron proyectos semejantes e incluso más ambiciosos. En Alemania, bajo la dirección de E. Sprockhoff, se iniciaba la publicación del Atlas der Megalithgräber Deutschlands ${ }^{18}$; A. S. Henschall editaba los dos tomos sobre los monumentos de Escocia ${ }^{19}$; y el "Centre National de la Recherche Scientifique, CNRS", publicaba los cinco primeros tomos del Inventaire général des megalites de la France, debidos a G. Cordier, J. Deprée, C. Leymarios,

Ricardo Batista Noguera, Sepulcros megaliticos de la Comarca del Moyanés, Prólogo de Luis Pericot. Barcelona. Instituto de Prehistoria y Arqueologia de la Diputación. 1961.

Ricardo Batista Noguera, Sepulcros megalíticos de la Comarca de Vic, Barcelona, Instituto de Prehistoria y Arqueologia de la Diputación, 1963

Luis Esteva Cruanas, Sepulcros megaliticos de las Gabarras (Gerona), I, prólogo de Luis Pericot, Gerona, Servicio de Investigaciones Arqueológicas de la Diputación de Gerona y Departamento de Prehistoria del CSIC., 1964; II, 1965; III, 1970.

i4 Miquel Cura Morera y Anna Maria Ferran Ramis, Sepulcros megaliticos de la Sierra de Roda (Alto Ampurdan. Gerona), Barcelona, Instituto de Arqueologia y Prehistoria de la Universidad, 1970.

is Miquel Cura Morena y Anna Ferran Ramis, Sepulcros megaliticos de la Vall de Cabó (Alto Urgel. Lerida). Barcelona, Instituto de Arqueologia y Prehistoria de la Universidad, 1971.

16. J. Padró. M. Cura y J. Abelanet, Sepulcros megalíticos de la Cerdanya y del Capcir. Barcelona. Instituto de Prehistoria y Arqueologia de la Diputación, 1975.

Luis Esteva Cruanas. Sepulcros megaliticos del Alto Ampurdan (Girona), Gerona, Instituto de Arqueologia y Prehistoria de la Universidad de Barcelona y Servicio de Investigaciones Arqueológicas de la Diputación de Gerona, 1979

${ }^{28}$ E. SPROCKHOOF. Atlas der Megalithgräber Deutschlands, 1, Schleswig-Holstein (Bonn 1986): 2. Mecklenburg-Brandenburg, Pommern (Bonn 1967); y 3. Niedersachsen-Westfalen (Bonn 1976).

19 A. S. Henshall, The Chambered Tombs of Scotland, 1 y 2 (Edimburgo, 1963 y 1972). 


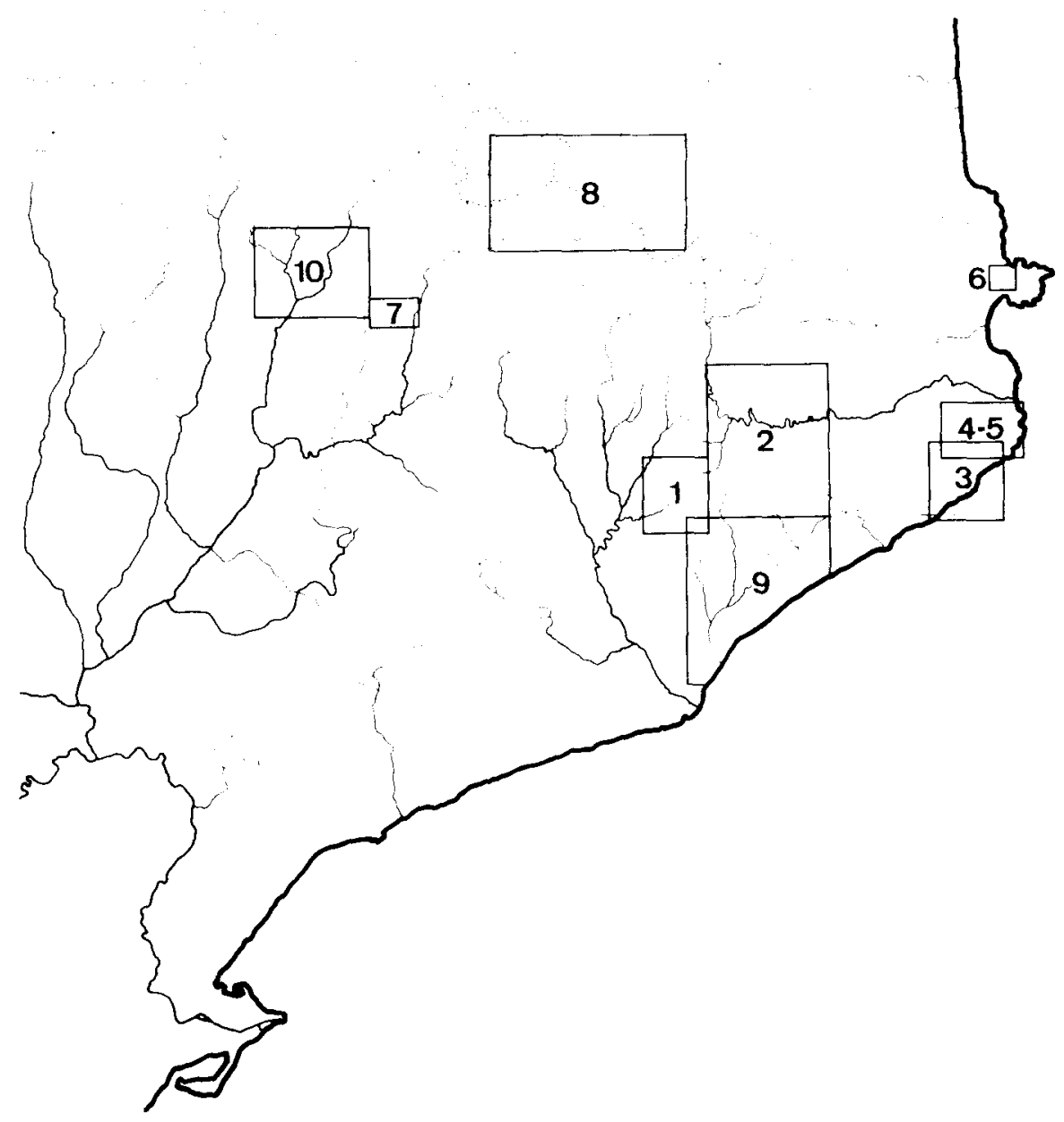

Figura 2. 1, Moianés; 2, Vic; 3, 4-5, Les Gabarres; 6. Serra de Roda; 7, Vall de Cabo; 8, Cerdanya y Capcir; 9, Barcelonés y Maresma; y 10, Alt Urgell.

J. Peek y Jean Clottes, todos ellos publicados como suplementos de la revista Gallia-Préhistoire ${ }^{20}$. Es de esperar que, en esta serie francesa,

* Gerard Condien, Inventaire des mégalithes de la France, 1, Indre-et-Loire, 1963, 132 págs., 40 figs. y XXXVIII láms.; Michel Gruet, 2, Maine-et-Loire, 1967, 345 págs., 97 figs. y VIII láms.; J. Desprié y C. LeYmarios, 3, Loir-et-Cher, 1974, 247 págs., 147 figs. y 19 láms.; J. PEEK, 4, Région parisienne, 1975, 408 págs., 38 figs. y 16 láms.; y Jean CLOTTES, 5. Lot, 1977, 552 págs., 185 figs. y 9 láminas. 
notable por su espléndida presentación, aparezca pronto algún tomo referente a las regiones pirenaicas.

Por lo que sabemos, en los últimos diez años no se ha sumado ninguna nueva aportación a los corpora de monumentos megalíticos de las regiones al $\mathrm{N}$ y al $\mathrm{S}$ de los Pirineos, lo que indudablemente es una sensible rémora para el avance científico y también para la protección de este patrimonio monumental. Seános permitido hacer un llamamiento para que este vacío sea progresivamente colmado.

Post scriptum. Cuando esta nota ya habia sido presentada al Congreso de Cervera, aunque sin verlo, hemos tenido noticia de un reciente trabajo dedicado a los dólmenes y menhires del Alt Empordà y del Vallespir oriental, que manifiesta un nuevo interés por el tema después de tantos años de atonía. Esta obra, por el número de monumentos recogidos -111 - da fe la conveniencia de las sistematizaciones que propugnamos ${ }^{21}$.

"AA. VV., Dolmens i menhirs: 111 monuments megalitics de lAalt Emporda $i$ del Vallespir oriental. Barcelona, 1988. 